\title{
Actinomyces israelii in the female genital tract: a review
}

\author{
D T P Evans
}

\section{Introduction}

In 198670 million women worldwide used the intra-uterine contraceptive device (IUCD) (see Part 2). Between 1.6 and $11.6 \%$ of IUCD users (see part 1) have infection with Actinomyces israelii (AI). Actinomyces is encountered from time to time in every sexually transmissible diseases clinic with periodic Papanicolaou (Pap) reports in IUCD users confirming the presence of AI. The issues of whether to treat with antibiotics or whether to remove the device or refer to the family planning clinic thus arise and confront the venereologist.

This review on $\mathrm{AI}$ is derived from journal articles spanning 64 years up to the present day (1992). The main body of the review is divided into four sections. Part 1 is concerned with actinomyces in the female genital tract, focusing on detection methods, prevalence and demographic and geographic variables. The next section is involved with the effect of IUCD insertion. Part 3 is concerned with pelvic infection due to actinomyces with emphasis on clinical presentation, diagnostic criteria and risk factors. Pelvic infection in this setting, it should be emphasised, is rare. The fourth part examines the management of the infected IUCD and of pelvic infection. Laparoscopy in diagnosis, the risk of pelvic actinomyces, prevention and modern management of the latter, feature amongst some of the final issues.

\section{Part 1}

Detection methods, prevalence and demographic, behavioural and geographic variables

The first report of the association of actinomyces-like organisms in the genital canals of women, employing the endocervical contraceptive device was by Barth in $1928 .{ }^{1}$ Actinomyces species are normal inhabitants of the oropharynx and possibly the bowel. ${ }^{2}$ Bhagavan $^{3}$ in 1978 identified actinomyces exclusively in patients wearing an intrauterine or vaginal foreign body (intra-uterine devices and pessaries).

Actinomyces are anaerobic or microaerophilic. Dilution of samples which usually contain other anaerobes, the use of media containing $2.5 \mathrm{mg} / 1$ metronidazole, or special selective media are useful in achieving successful isolation. Cultures are often unsuccessful because the technique is poor, and this has persuaded many American workers to rely solely on microscopy of stained smears of material, sometimes confirming their impression by using direct immunofluorescent techniques. With the very wide range of coryneforms and lactobacilli present in the vaginal flora, the specificity of such techniques is critically important if they are used as the sole means of diagnosis. It is probable that such differences underly a controversy as to whether actinomycetes are normal members of the vaginal flora. ${ }^{4}$

Further work is needed to investigate the identity of the microcolonies, seen on Pap smears, that are not Actinomyces israelii and to confirm or refute whether actinomycetes such as Actinomyces propionica can be grown from the vaginas of patients without an IUCD in situ. It seems proper to regard these actinomycetes as part of the normal flora of patients with an IUCD in situ. ${ }^{4}$ Actinomyces has been found, however, in Dybdahl's 1991 series $^{5}$ in a tiny percentage (2 of 17,734 cervical smears) of those without an intra-uterine device in situ (the latter represents $88 \%$ of the total); thus $12 \%$ of the women had an IUCD. However, in 1983 Persson et al ${ }^{6}$ stated that Actinomyces israelii was part of the indigenous genital flora. Dybdahl regarded actinomyces colonisation of the female genital tract as a rare event in women who are not users of IUCDs.

Demographic and Geographic Variables and Prevalence Early reports of pelvic actinomyces can be found in the literature in 1979 in Canada. ${ }^{7}$ The vast majority of reports of actinomyces internationally over the years have been in intra-uterine contraceptive device (IUCD or IUD) users. The earliest (1982) and biggest study of women screened for AI by cervical Papanicolaou smears comes from the USA. ${ }^{8}$ The study was of 69,925 smears and the organism was not identified in non-IUCD users. The prevalence of $\mathrm{AI}$ among IUCD wearers ranged from $16 \%$ (general population) to $5 \%$ (clinic population).

In India the prevalence rate for actinomyces in 1986 for 815 IUCD users was $6.99 \% .{ }^{9}$ In India a year earlier Nayar et al found a $2 \cdot 8 \%$ prevalence of actinomyces in 350 cases using an IUCD. This was with pap smears confirmed by immunofluorescence. ${ }^{10}$ This pattern was maintained in Sweden ${ }^{6}$ and in Kuwait when AI was recovered only from IUCD users. ${ }^{11}$ In 1989 in Australia ${ }^{12}$ in IUCD users $11.6 \%$ of 973 cases had infection with AI, the majority of whom were asymptomatic. ${ }^{20}$

Lastly in Denmark in 1991 it was found that $8.6 \%$ of IUCD users had actinomyces. Thus, there are reports of actinomyces in association with IUCDs from North America, 
Scandinavia, Middle East, Australia and Asia. There is every reason to suspect that its ubiquitous in association with IUCDs and probably very rarely present worldwide in those without IUCDs.

Ethnic and behavioural differences in susceptibility to actinomyces are issues that have not been addressed in the literature. For example, there have been no studies of differences between blacks and whites in America.

Detection methods Three species of actinomyces, $A$ israelii, $A$ naeslundi, and $A$ odontoyticus, and a close relative Arachnia propionica are relevant to discussion of actinomycetes in the human genital tract. These bacilli are characteristically gram-positive and branching but can apparently occur as single bacilli looking not unlike coryneforms. ${ }^{4}$

Pine et al in $1981^{13}$ set out to determine whether actinomyces or arachnia species were common in the vaginas of apparently healthy women. They used a staining procedure in which pepsin digestion and rhodamine conjugate of normal rabbit globulin are used to reduce non-specific staining by fluorescein conjugated antibody and the application of this procedure to demonstrate $A$ israelii, $A$ naeslundi and Arachnia propionica in cervicovaginal smears taken from apparently healthy women who had worn or who had not worn an IUCD. Earlier workers, including Gupta ${ }^{14}$ failed to find a smear positive for Actinomyces naeslundi (AN).

In later studies Gupta and collaborators identified $\mathrm{AI}$ in cervicovaginal smears by using fluorescein isothiocyamate (FITC) conjugates of species-specific globulins. Ultimately, actinomycete-like organisms were observed in stained smears from 520 women: in 250 out of 266 of these patients, AI was observed by staining with the FITC conjugate. ${ }^{13}$

Further diagnostic studies were performed using precipitin test antibodies against AI. ${ }^{615}$ In 1984, Persson and Holmberg used the latter system, comprising a combination of counter immunoelectrophoretic and crossed immunoelectrophoretic gel techniques. The specificity of the test system for the detection of cases of genital actinomyces was $98 \%$ and the sensitivity was $83 \%$. The accuracy was $100 \%$ for negative prediction and $45 \%$ for positive prediction. Thus the test was shown to be valuable for a non-invasive diagnosis of genital actinomyces.

The same authors' second study in $1985^{6}$ was to detect a local antibody response to $\mathrm{AI}$ in uterine secretions of 100 women. There was an analysis by counterimmunoelectrophoresis for the occurrence of precipitation reactions against AI antigens. Precipitation reactions were found in secretions from seven women and they correlated with the long term use of plastic intrauterine devices. The precipitating components were not immunoglobulins, and their importance for AI colonisation and in disease development needs to be further investigated.

Leslie and Garland, from Melbourne, in
1991,16 compared direct immunofluorescence (IF) with traditional culture for the detection of AI. In a smaller sample than that of Cleghorn and Wilkinson, they examined endocervical and IUCD smears from 124 IUCD users of 11 specimens that gave positive results by immunofluorescence, only one was positive by culture. Thus they agree with Valicentis's findings 9 years previously. ${ }^{8}$ This study ${ }^{16}$ however, crucially involved patients whose IUCDs had been in situ for less than 2 years, a significant difference from the other study. Thus, they would tend to favour immunofluorescence as the appropriate method. These workers criticised culture techniques as being insensitive, because of the oxygen-sensitive, fastidious and slowgrowing nature of the organisms and difficulty in selecting them from other faster-growing anaerobes.

Examination of Pap-stained smears for AI is more sensitive than culture, but still lacks overall sensitivity and specificity. By morphology alone AI, other related actinomyces, arachnia and nocardia are all indistinguishable: so this technique has its faults.

Overall direct immunofluorescence using fluorescein isothiocyanate labelled antisera against $\mathrm{AI}$, is best and most sensitive and specific, although in some long term IUCD users (more than 36 months), culture and IF may be equally good for diagnosis.

A recent study by Dybdhal ${ }^{5}$ utilised old fashioned morphology as the means of identification of actinomyces as used by Duguid, 17 and Nayar, ${ }^{10}$ in 1985. Such traditional methods still have their advocates. Actinomyces was defined as the presence of dark, dense aggregates of uniformly sized filaments revealing distinct, acute-angle branching and radiation from a central core. ${ }^{5}$

\section{Part 2}

Effect of IUCD insertion (with reference to type of device and duration of its usage)

IUCD use involved 70 million women worldwide in 1986 . In India 5.8 million women were fitted with IUCDs in the years 19811986.9

Valicenti states $^{8}$ that an IUCD has been shown to induce a mild inflammatory response in the endometrium, with focal necrosis. This may produce an environment of anaerobiosis that encourages growth of $A$ israelii and other anaerobic organisms. In his series of 112 cases in those patients whose IUCDs were removed and not replaced, subsequent Pap smears failed to reveal actinomyces organisms. It could be concluded that in the vast majority of cases, the organism causes a superficial infestation of the endometrium, which is associated with the menstrual period. This is in contrast to true infection with associated tissue invasion.

Pine ${ }^{13}$ observed the presence of AI, $A$ naeslundi, and Arachnia propionica in cervicovaginal smears from women, who did use and, who did not use an IUCD AI was commoner in women using an IUCD but, there was no evidence that the use of an IUCD 
influenced the presence of either $A$ propionica or $A$ naeslundi (AN).

Bonnez in 1985 was the first to demonstrate AN as a causative agent of pelvic actinomyces in the presence of an IUCD. ${ }^{18}$ In the 1982 study of Valicenti, the 212 IUCD users who had AI had an average of $6 \cdot 1$ years of IUCD usage. ${ }^{8}$ The types of IUCD were as follows:

Type
Lippes Loop
Dalkon Shield
Saf-T-Coil
Unknown
Tatum-T
Cu-7

$\begin{array}{cc} & \text { Average duration } \\ \% & \text { (years) } \\ 36 & 7 \cdot 6 \\ 20 & 4 \cdot 4 \\ 20 & 5 \cdot 1 \\ 15 & 6 \cdot 3 \\ 8 & 3 \cdot 3 \\ 1 & 2 \cdot 0\end{array}$

Nayar et allo discovered that the time of insertion of an IUCD (postpuerperal postmenstrual or after medical treatment of pregnancy) did not show any correlation with the presence of actinomyces infection. Duguid et al 17 emphasised that a significantly greater colonisation of actinomyces-like organisms are found in women with plastic devices, when compared with copper device users. Persson ${ }^{15}$ alludes to this predisposition for plastic. The antibacterial effect of copper is known, ${ }^{19}$ and reports have shown that following insertion, such devices release about 27 micrograms of copper daily and this amount decreases with time. ${ }^{11}$ It may merely be a reflection of the fact that copper IUCDs are changed more frequently than the plastic ones.

Data from India ${ }^{9}$ suggest that prolonged use (more than 2 years) of an inert or copper IUCD promotes the overgrowth of actinomyces in the vagina and, in turn, this can be detected by routine cervical cytology. 8920

A fairly recent report (1989) showed that the incidence of $\mathrm{AI}$ in the female genital tract of IUCD users was more than doubled when the IUCD was worn for longer than 4 years. The type of IUCD worn did not appear to be an important risk factor. ${ }^{12}$ Local fashions appear to govern type and popularity of devices. ${ }^{8}$

Cleghorn and Wilkinson ${ }^{12}$ showed that immunofluorescent staining of cervical smears identified $64 \%$ of cases of AI. Specific culturing for AI or cytological screening of Pap smears, in addition, were required to increase the isolation rate to nearly $90 \%$. The data in this paper support the view that IUCDs should be changed every 4 years. Only the Dalkon Shield and Saf-T-Coil, amongst all IUCDs were associated with a statistically significant detection rate of $\mathrm{AI}$ in this study. ${ }^{12}$ Other IUCD types were:- Lippe S Loop; Grafenber Ring; Copper 7; Gynae-T (Copper T); Multiload (Copper 250). ${ }^{12}$

Cleghorn and Wilkinson showed that for diagnosis, culture for actinomyces was more reliable when the IUCD had been in place for more than 36 months, suggesting that these were more advanced cases. Immunofluorescence of cervical smears for AI is most useful for short term IUCD users, when culture is less reliable. Cytology was the least sensitive technique, and no cases of actinomyces were detected when the IUCD had been worn for less than 12 months.

\section{Part 3}

Incidence of pelvic infection due to actinomyces. Clinical presentation, diagnostic criteria and risk factors

There is a need for careful differentiation between the presence of AI of the female genital tract and pelvic actinomycotic disease. The latter is very rare.

Valicenti ${ }^{8}$ stated that sporadic case reports have implicated this organism as the aetiologic agent in salpingo-oophoritis, tuboovarian abscess, and endometritis in women using IUCDs. Because the morphology of the actinomycete may be variable and dependent on growth conditions, $A$ israelii often is confused with other Gram-positive, non-sporeforming bacilli and non-related bacteria such as clostridia, corynebacteria, listeria, and leptotrichia. Actinomycetes are not cultured or isolated readily from cervical material using basic anaerobic media, which makes confirmation difficult. Direct immunofluorescence using fluorescein isothiocyanate-labelled antisera against $A$ israelii is sensitive and specific and has become the method of choice for positive identification.

Bhagavan and Gupta $^{3}$ stated that the symptoms and signs of actinomyces in the genital tract of IUCD users, which could possibly be the harbinger of more serious pelvic actinomycosis. In this series there were: vaginal discharge in 17; pelvic pain in 4 ; pelvic and abdominal pain in 2; menorrhagia/ intermenstrual bleeding in 3 ; fever in 2 ; pelvic mass in 2 ; history of pelvic inflammatory disease (PID) in 3.

Cleghorn and Wilkinson 12 found that the detection of $A$ israelii in the female genital tract was associated with an almost four-fold increase in the incidence of PID indicating that colonisation may progress to an infection in a small number of women in the endometrium and fallopian tubes under appropriate conditions of tissue damage. This is controversial see start of section 2 . At present there is no way of predicting which IUCD patients who are colonised with $\mathrm{AI}$ will subsequently develop serious pelvic infection.

Leslie and Garland stated in 1991 that reports of actinomyces species in cases of PID in IUCD users has varied from $17 \%$ to $25 \% .{ }^{16}$ In association with this it must be borne in mind that the prevalence of actinomyces carriage in IUCD users (as implied in part 1) varies from $1.6 \%$ to $11.6 \%$ in different series.

Spread of AI within and without the genital tract is uncommon, but when it occurs, the right ovary and fallopian tube are most frequently affected, with or without parametrial disease. ${ }^{7}$ The uterus appears to be relatively resistant to infection and endometrial involvement is extremely rare. ${ }^{2}$

O'Brien in $1975^{7}$ reported a case of endometrial infection with AI associated with an IUCD, and presence of $\mathrm{AI}$ in inflamed 
omental and pericolic tissues. The staining reactions of $\mathrm{AI}$ were present in tissue adherent to the IUCD, that is not Ziehl-Neelson acid-fast, yet positive for Grocott's modification of Gomori's methenamine silver nitrate stain. Also, radially arranged, gram positive, beaded, branching filaments, typical of AI were observed in the specimen.

Indeed pelvic actinomyces in 1976 was considered one of the rarer diseases confronting the gynaecologist, with only 300 cases reported then. ${ }^{20}$ It is insidious in onset and clinically indistinguishable from other forms of PID. However, in 1976 anaerobic culture and histological analysis of the surgically treated pelvic abscess had a high failure rate for identification of the organism. ${ }^{20}$ Lomax et al presented four cases of actinomyces involving the uterus and adnexal structures collected over a 20 year period (1954-1974) at the University of Virginia. In two patients IUCDs were associated with endometrial actinomycosis and adnexal abscesses.

The difficulty encountered in identifying AI indicates that the infection is often undetected (pelvic actinomycosis is usually confined to the tubes, ovaries, uterus and parametrium. Tubo-ovarian abscesses are part of the spectrum). Hence the value of Pap cervical smears for early detection and prophylactic treatment before AI has been well established (see below). Gallium 67 and indium scans are helpful in localising late disease, that is occult abscesses as suggested by Lomax et al. ${ }^{20}$ Laparoscopy has its place (see Part 5) as does ultrasound of ovaries and pelvis. Magnetic resonance imaging and computed tomography of the abdomen and pelvis could be useful in diagnosis of focal abscesses. Lastly culdocentesis is an adjunct to diagnosis.

Treatment of pelvic actinomyces, as recommended by Lomax, is a combination of high doses of penicillin and chloramphenicol to treat the anaerobes. Oral penicillin should then continue for at least 2 months.

Cohen and Silberberg in 1976 reported a case of an extensive tubo-ovarian abscess caused by AI. They warn that when chronic PID does not respond to therapy, when the sedimentation rate remains high, and the patients general debility is progressing, an actinomycotic infection should be suspected. Their therapeutic advice was to extirpate the infected tissue in combination with penicillin. ${ }^{22}$ This could include salpingooophorectomy and hysterectomy.

In 1976 the reported locations of all known cases of pelvic actinomyces were reviewed. ${ }^{22}$ Cases were recorded at the time of diagnosis. Whether medical treatment had been given or not was immaterial. These essentially were abscesses or 'foci' of infection. Thus there were, up to 1976, 66 cases of actinomycotic tubo-ovarian abscesses in the world literature. Eighteen were in the fimbria on the right, nine were in the fimbria on the left, 18 were in the ovary on the right, 21 were in the ovary on the left, seven were in the uterus, 12 were in the adnexa on the right, 12 were in the adnexa on the left, nine were in the parametrium on the right, nine were in the parametrium on the left.

There is a need for sophisticated anaerobic culture from female pelvic infection or abscesses; culture from female pelvic infection or abscesses; culture for actinomyces requires prolonged incubation. ${ }^{23}$

\section{Part 4}

Management of infected IUCD and of pelvic infection

Valicenti et al ${ }^{8}$ recommended conservative management for asymptomatic patients, with cytologically detected AI, to include removal of the IUCD, and repeated Pap smear after the next menstrual period. Subsequent investigation invariably fails to identify actinomyces-like organisms. The organism may cause a superficial endometritis, which is shed with the menstrual period: this appears to be a very satisfactory approach.

There is common agreement that in PID with actinomyces infection, removal of the IUCD and antibiotics are mandatory. When actinomyces are present without any evidence of PID, some operators would treat conservatively and not remove the IUCD. 5 The vast majority would, however, in this circumstance, remove the IUCD and repeat the Pap smear after the next menstrual period. ${ }^{8}$ Should it be negative, some might replace it with a fresh IUCD.

The views of Cleghorn and Wilkinson ${ }^{12}$ are pertinent here. They state that there is no way of predicting which IUCD patients, who are colonised with $\mathrm{AI}$, will subsequently develop serious pelvic infection. Therefore, all IUCD users, with symptoms of possible PID, should be screened for AI so that antibiotic treatment and/or IUCD removal can be carried out, to prevent the colonisation progressing to pelvic infection.

Leslie and Garland ${ }^{16}$ in 1991 reminded us that management of IUCD users with actinomyces is arbitrary and varies from centre to centre. They advocate 2 yearly immunofluoresence tests, of a cervical smear, and if asymptomatic actinomyces carriage is discovered, removal of the device. Earlier cervical smears would be done if the patient was symptomatic.

If the IUCD user presents with localised symptoms, then amoxycillin $500 \mathrm{mgs}$ tds and metronidazole $400 \mathrm{mgs}$ bd or tds doxycycline $100 \mathrm{mgs}$ bd for 2-3 weeks are given and the device is removed. Pelvic actinomyces is a potentially life threatening disease but fortunately rare.

If a pelvic mass is present, aggressive and prolonged antibiotic therapy plus possible surgery is required (see part 3 ). Sexually Transmissible Disease Clinics becoming "Sexual Health Centres" with Family Planning expertise may, in the future, more often take part in the removal of IUCDs. Of course, there would be simultaneously monitoring of the contraceptive wishes of the 
patient and this is the overriding consideration in this whole debate.

Part 5

How pelvic actinomycosis resembles and differs from other forms of PID

The incidence of PID, in general, is reported to be higher in IUCD users than in nonusers, although the type of IUCD, patient selection, and the presence of sexually transmitted organisms are important factors in assessing the real risk. ${ }^{16}$

Vessey et al reported hospital admission rates in the United Kingdom with a diagnosis of acute PID to be 1.51 per 1000 women for IUCD users compared with $0 \cdot 14 / 1000$ for non-users. They found the Dalkon Shield (now withdrawn) to be associated with the highest risk and copper containing devices to be associated with the lowest risk. ${ }^{24}$

Recent observations appear to indicate a cause and effect relationship between use of IUCDs and pelvic actinomycosis. The relationship between presence of actinomyceslike organisms in the cervical smears of IUCD users, and the subsequent development of PID, is more difficult to establish, because the majority of such cases are asymptomatic at the time of smear collection and may remain so for a considerable length of time. ${ }^{9}$

It was formerly thought that the lactinomyces route of entry in PID and mode of spread was via local lymphatic pathways from the gastrointestinal tract and haematogenous pathways during systemic infection. ${ }^{3}$ The reality is an ascending route of infection via the IUCD. ${ }^{3}$ This route of infection in actinomycotic PID is the same as for PID in general; namely upward spread through the cavities of the cervix, uterine body and tubes. ${ }^{24}$

Laparoscopy appears to be a useful method to diagnose AI in PID in general. Brihmer from Sweden in $1987^{26}$ laparoscoped 359 patients to verify the diagnosis of salpingitis. PID was found in $52 \%$ of the cases. Bacteriological cultures from the fimbrial lumen and/or pouch of douglas revealed AI in 3\% compared with Chlamydia trachomatis in $12 \%$ and bacteroides in $5 \% .26$

Symptoms and signs criteria for inclusion in this study were symptoms of less than 3 weeks duration of low abdominal pain, vaginal discharge and tenderness at gynaecological examination. ${ }^{26}$

The usefulness of laparoscopy is demonstrated by the fact that completely normal inner genitalia were found in more than one third of the cases. Irregular bleeding, an ESR above $15 \mathrm{~mm}$ in 1 hour and temperature above $38^{\circ} \mathrm{C}$ in this study constituted significant signs for the diagnosis of salpingitis in general. Whether this relationship and predictive valve holds for AI PID alone is not known.

Large series of laparoscopically verified cases of AI in PID need to be studied in detail to answer such questions as to how the symptom and sign profile of such patients, differs if at all from classical non AI cases of PID. Do AI cases of PID have the same complications profile as PID in general? The answers are not known. Long-term infertility, ectopic pregnancy and chronic pelvic pain are all in question.

The risk of pelvic actinomyces: a summary

How may pelvic actinomycosis be prevented? Perhaps long-term penicillin or doxycycline could be tried in IUCD users. Long-term amoxycillin or flagyl are not practicable. The simple answer is to change the IUCD every 4 years. It should be suspected in any long-term IUCD user. How is it diagnosed? It is diagnosed by a Pap smear backed up by culture and immunofluorescence. Laparoscopy or CT are adjuncts. Advanced cases may require laparotomy (see previous sections).

\section{Modern management}

Most authors agree that for a small or moderate size pelvic abscess (up to $8 \mathrm{~cm}$ maximum diameter), medical treatment is worth a trial.27 It would appear that penicillin or another beta lactam antibiotic, aminoglycoside and an agent such as metronidazole, highly active against anaerobes, should all be used together. Careful monitoring of the clinical state of the patient is needed, including if possible ultrasound assessment of the size of the abscess. If any worsening occurs, or if distinct improvement in her condition is not apparent within $48-72$ hours of starting treatments, then surgical treatment is needed.27 All studies report a very poor fertility prognosis after antibiotic treatment for pelvic abscess complicating PID.

Recently an international team ${ }^{28}$ have reviewed the World Health Organisation's IUCD clinical trial data to explore the incidence and patterns of PID (in general as opposed to specifically $\mathrm{AI}$ induced) risk with use of an IUCD. After adjustment for confounding factors PID risk was more than six times higher during the 20 days after insertion than during later times. The findings indicated that PID among IUCD users is most strongly related to the insertion process and to background risk of STDs. The authors state that IUCDs should be left in place up to their maximum lifespan and they do not focus on the long-term potential actinomyces risk. So, in conclusion, based on these data, and the findings in this review, PID risks are at the start and the end of the life of the IUCD.

Lastly here is the devil's advocate in this review:- Is there good evidence that actinomycosis is a primary pathogen, or does it occur as a complication of some common causes of PID, because of inadequate treatment for anaerobic secondary infection? The simple answer to this question is that it is not known; however, Leslie and Garland ${ }^{16}$ expand on this issue. They say that in most cases PID is polymicrobial, with various aerobes and anaerobes in addition to sexually transmitted organisms, the commonest of which is Chlamydia trachomatis. 


\section{Conclusions}

The process of colonisation of an IUCD by AI is slowly progressive, since there is little change in rate of AI detection before a IUCD has been in situ for $\mathbf{4 8}$ months. The incidence is increased to a maximum of over $30 \%$ colonisation of IUCD by AI after 8 years.

Immunofluorescence and culture are the best methods of detection of AI.

There is a need to try and determine which IUCD wearers are colonised with A.I., and subsequently develop serious pelvic infection.

Plastic IUCDs are more likely to be colonised by actinomyces like organisms than copper devices.

AI in the female genital tract of IUCD users more than doubles (in incidence) when the IUCD is worn for longer than 4 years. For this reason it is prudent to change IUCDs every four years. Pelvic actinomyces is indistinguishable from other forms of PID. All IUCD users with symptoms of possible PID should be screened for AI so that appropriate treatment can be given. Laparoscopic bacteriological culture from the fimbrial lumen is the best method of confirmation of AI pelvic inflammatory disease.

PID is mostly polymicrobial with a combination of various aerobes and anaerobes.

Plans for treatment of AI: In asymptomatics with actinomyces, remove IUCD and possibly replace later if certain conditions are satisfied. With asymptomatics it is necessary to review the contraceptive wishes of the patient, before putting another IUCD back in situ. With all PID cases, the IUCD must be removed, followed by antibiotic treatment.

It is questionable whether the IUCD should be left in place in asymptomatics, and also whether to leave the IUCD in place with antibiotic "cover" in PID.

For pelvic actinomycosis treatment it is prudent to use a surgical approach for tuboovarian abscesses greater than $8 \mathrm{~cm}$ diameter and to reserve medical treatment for the rest.

It seems to be established that actinomyces can be grown from the vaginas of patients without an IUCD in situ. Thus, we have no need to adhere to the belief that $\mathrm{AI}$ is not a commensal in the vagina, but is introduced there via oral sex.

I thank Lorraine Cleary for her considerable patience with typing the manuscript and drafts.

1 Barth H. Uber Paramatritis Actinomycotia und Ihre Enststenhung Arch Gynaekol 1928;134:310-21.
2 Luff RD, Gupta PK, Spence MR, Frost JK. Pelvic actinomycosis and the intrauterine contraceptive devices. $f$ Clin Pathol 1978;69:581-6.

3 Bhagaven BS, Gupta PK. Genital actinomycosis and intrauterine contraceptive devices. Human Pathology 1978;9:567-78.

4 Warren ER. Normal flora of the vagina Chap. 2. In: Hare MJ. Genital Tract Infection in Women. Edinburgh, Churchill Livingstone, 1988.

5 Dybdahl $\mathrm{H}$, Hastrup J, Baandrup U. The clinical significance of actinomyces colonisation as seen in cervical smears. Acta Cytol 1991;35:142-3.

6 Persson E, Holmberg K. Study of precipitation reactions to Actinomyces israelii antigens in uterine secretions. $\mathcal{f}$ to Actinomyces israelit antigens

7 O'Brien PK. Abdominal and endometrial actinomycosis associated with an intrauterine device. $\mathcal{F} \mathrm{Can} \mathrm{Med} \mathrm{Assoc}$ 1975;112:596-7.

8 Valicenti JF, Pappas AA, Graber CD, Williamson HO, Willis NF. Detection and prevalence of IUD associated actinomyces colonisation and related morbidity. $\Im A M A$ 1982;247:1149-52.

9 Mali B, Joshi JV, Wagle U, et al. Actinomyces in cervical smears of women using intrauterine contraceptive devices. Acta Cytol 1986;30:367-71.

10 Nayar M, Chandra M, Chitraratha K, Das SK, Chowdhary GR. Incidence of actinomycetes infection in women using intrauterine contraceptive devices. Acta Cytol 1985;29:111-6.

11 Elhag KM, Bahar AM, Mubarak AA. The effect of copper intrauterine contraceptive device on the microbia ecology of the female genital tract. $\mathcal{F}$ Med Microbio 1988;25:245-51.

12 Cleghorn AG, Wilkinson RG. The IUCD associated incidence of Actinomyces israelii in the female genital tract. Aust N.Z. $\mathcal{F}$ Obstet Gynaecol 1989;29:445-9.

13 Pine L, Malcolm GB, Curtis EM, Brown JM Demonstration of actinomyces and arachnia species in cervicovaginal smears by direct staining with speciesspecific fluorescent antibody conjugate. $¥$ Clin Microbiol 1981;13:15-21.

14 Gupta PK, Erozan YS, Frost JK. Actinomycetes and the IUD; an update. Acta Cytol 1978;22:281-2.

15 Persson E, Holmberg K. Clinical evaluation of precipitin tests for genital actinomycosis. $\mathcal{f}$ Clin Microbiol 1984; 20:917-22.

16 Leslie DE, Garland SM. Comparison of immunofluorescence and culture for the detection of Actinomyces israeli in wearers of intrauterine contraceptive devices. $\mathcal{F} \mathrm{Med}$ Microbiol 1991;35:224-8.

17 Duguid HLD, Parratt D, Traynor R. Actinomyces like organisms in cervical smears from women using IUCDs. $B M F$ 1980;281:534.

18 Bonnez W, Lattimer G, Navaratnasingam A, Mohanraj C, Johnson TH. Actinomyces naeslundii as an agent of pelvic actinomycosis in the presence of an intrauterine device. actin Microbiol 1985;21:273-5.

19 Woler SM, Rolla G. Comparison between plaque inhibiting effect of chlorhexidene and aqueous solution of copper and silver ions. Scand F Dematol 1982;90:131-3.

20 Lomax CW, Harbert GM, Thornton WN. Actinomycosis of the female genital tract. Obstet Gynecol 1976;48: 341-6.

21 MacCarthy J. Actinomycosis of the female pelvic organs with involvement of the endometrium. $\mathcal{F}$ Pathol Bacteriol 1955;69:175.

22 Cohen A, Silberberg B. Pelvic actinomycosis Int $\mathcal{f}$ Gynaecol Obstet 1976;14:241-5.

23 Hansen LK. Bilateral female pelvic actinomycosis. Acto Obstet Gynaecol Scand 1989;68:189-90.

24 Hare MJ. Pelvic inflammatory disease Chap 6 In: Hare MJ. Genital Tract Infection in Women. Edinburgh, MJ. Genital Tract Infection in

25 Hare MJ. Pelvic inflammatory disease Chap 6 In: Hare MJ. Genital Tract Infection in Women. Edinburgh, Churchill Livingstone. 1988;134.

26 Brihmer C, Kallings I, Nord CE, Brundin J. Salpingitis aspects of diagnosis and etiology; a 4 year study from a Swedish capital hospital. Eur $\mathcal{f}$ Obstet Gynaecol Reprod Biol 1987;24:211-20.

27 Hare MJ. Pelvic inflammatory disease. Chap 6 In: Hare MJ. Genital Tract Infection in Women. Edinburgh, Churchill Livingstone. 1988;153.

28 Farley TMM, Rosenberg MJ, Rowe PJ, Chen JH, Meirik $O$. Intrauterine devices and pelvic inflammatory disease: an international perspective. Lancet 1992;339:785-8. 